\title{
Research on the function and related mechanism of $P 27$ gene in the intervertebral disc degeneration of mice
}

\author{
XIAO LIU ${ }^{1,2,5}$, DEGUO WANG ${ }^{1,3}$, ZHENGZHENG ZHANG $^{4}$, FENGHUI ZHU ${ }^{2}$, \\ AIMING YAO $^{2}$, JIWEI TIAN ${ }^{1}$ and DENGSHUN MIAO
}

\begin{abstract}
${ }^{1}$ Department of Orthopedics, Shanghai General Hospital of Nanjing Medical University, Shanghai 200080;
${ }^{2}$ Emergency Center, Affiliated Hospital of Xuzhou Medical University, Xuzhou, Jiangsu 221002; ${ }^{3}$ Department of Orthopaedics, Shanghai Songjiang District Central Hospital, Shanghai 201600; ${ }^{4}$ Department of Obstetrics and Gynecology, Affiliated Hospital of Xuzhou Medical University, Xuzhou, Jiangsu 221002; ${ }^{5}$ The Research Center for Bone and Stem Cells, Department of Anatomy, Histology and Embryology, Nanjing Medical University, Nanjing, Jiangsu 211166, P.R. China
\end{abstract}

Received March 24, 2017; Accepted June 2, 2017

DOI: 10.3892/etm.2017.4584

\begin{abstract}
This study aims to investigate the function and related mechanism of $P 27$ gene in intervertebral disc (IVD) degeneration of mice. X-ray, immunohistochemical staining, and alkaline phosphatase (ALP) histochemical staining were used to analyze the phenotypic difference of the intervertebral discs of 4-week-old mice with P27 gene knockout (P27-- $)$ and wild-type (WT) mice in the same brood. Protein in the intervertebral disc was extracted and western blot analysis was employed to detect the changes in the expression of related molecules in the Shh-signal pathways, including Shh, Patched, Smoothened and Gli2. As a result, the ALP histochemical staining revealed that the ALP-positive area of mice in the P27-- group was obviously increased compared to the 4-week-old mice of the same brood in the WT group. In addition, the Col-I immunohistochemical staining showed that the Col-I-positive area of mice in the $\mathrm{P} 27^{-/}$group was significantly increased compared to mice in the WT group. Furthermore, Smo-positive cell rate of mice in the P27/- group was apparently increased compared to mice in the WT group. Western blot analysis revealed that in terms of changes of protein expression levels of Shh, Patched, Smoothened and Gli2 in the intervertebral disc, protein expression levels of Shh, Patched, Smoothened and Gli2 of mice in the $\mathrm{P} 27^{-1}$ group were significantly increased compared to those of mice in the WT group. The results show that P27 defi-
\end{abstract}

Correspondence to: Dr Jiwei Tian, Department of Orthopedics, Shanghai General Hospital of Nanjing Medical University, 100 Haining Road, Shanghai 200080, P.R. China

E-mail: tjw9060@163.com

Dr Dengshun Miao, The Research Center for Bone and Stem Cells, Department of Anatomy, Histology and Embryology, Nanjing Medical University, 101 Longmian Avenue, Jiangning, Nanjing, Jiangsu 211166, P.R. China

E-mail:dsmiao@njmu.edu.cn

Key words: P27, mice, intervertebral disc degeneration ciency activates the expression of Shh-signal pathway and promotes the proliferation of osteoblast, thus, playing a role in promoting IVD degeneration, which provides a scientific and reliable experimental basis for the treatment of the IVD degeneration-related diseases in clinical practice.

\section{Introduction}

Intervertebral disc (IVD) degenerative disease is a common disease in clinic which is also the major factor of lumbago $(1,2)$. Currently, the main methods used to treat these diseases in clinic are nucleus pulposus removal, spinal canal decompression and degenerative spinal segmental fusion. However, these methods only relieve patient symptoms for a short period of time and their long-term efficiency is unsatisfactory, and they may cause multiple complications (3-5). Therefore, identifying a new target for the treatment of intervertebral disc degenerative diseases in order to perfect the clinical treatment strategy for the IVD degeneration is required.

Previous findings showed that the Shh-signaling pathway plays an important role in the process of the intervertebral disc development, differentiation and degeneration (6). Furthermore, the $P 27$ gene is a significant gene in the regulation of the Shh-signal pathway $(7,8)$. Shimura-Miura et al (9) cultured a 13-week-old male Sprague Dawley (SD) rat at fasting status for 6 and $48 \mathrm{~h}$ after the intervertebral disc nucleus pulposus cell, to induce cell senescence, and found that P27kip1 expression was elevated in nucleus pulposus cell, and cell percentage was significantly increased at G0/G1 stage, while apparently decreased at S stage, indicating that P27kip1 may be involved in the development process of IVD by regulating the cell cycle. To the best of our knowledge, there is currently no study showing that the $P 27$ gene plays a role via the Shh-signaling pathway. On the basis of the successful establishment of mouse P27 gene knockout (P27 $7^{-1}$ ) of the IVD degeneration model, this experiment primarily investigates the role of $P 27$ gene in the development process of IVD and determines whether $P 27$ gene played a role via the Shh-signaling pathway. Subsequently, the pathogenesis and possible related molecular mechanism of IVD were further revealed genetically 
to provide an experimental and theoretical basis for the early prevention and treatment of IVD degeneration.

\section{Materials and methods}

Experimental animal. P27 $7^{-/}$IVD degeneration model of mice was established by the Animal Experiment Center of Shanghai General Hospital of Nanjing Medical University. Male and female mice were taken out for mating, and genotype identification was performed for their offspring. The 4-week-old wild-type (WT) mice (WT group, $\mathrm{n}=36$ ) and $\mathrm{P} 27^{-/}$mice (P27 $7^{-/}$group, $\left.\mathrm{n}=36\right)$ in the same brood were obtained. Mice were housed in a temperature controlled room $\left(21 \pm 2^{\circ} \mathrm{C}\right)$ on a 12:12-h light/dark cycle (lights on at 06:00 a.m.). All mice had free access to water and food. This study was approved by the Animal Ethics Committee of Nanjing Medical University Animal Center.

Material drawing. The mice were sacrificed by the cervical dislocation when they were 4 weeks old. IVD degenerative vertebral bodies of mice were dissected, separated and $4 \%$ of paraformaldehyde was utilized to fix them overnight. Conventional dehydration and paraffin embedding were employed. The bodies were sectioned for standby application.

$X$-ray examination. Prior to sacrifice, at the 4 th week, X-ray examination was conducted. The experimental mice inhaled isoflurane and were anesthetized transitorily, and an X-ray examination was carried out using a metal needle to guide by positioning the location of IVD in the mouse's tail. The changes of the IVD height were observed, and then the intervertebral disc height index (DHI) was calculated.

Immunohistochemical staining. First conventional deparaffinage was performed for paraffin sections and then immunohistochemical staining was carried out for them, followed by observation under a light microscope (BX-42; Olympus, Tokyo, Japan).

Alkaline phosphatase (ALP) histochemical staining. After paraffin sections were processed by conventional deparaffinage and hydration, $1 \% \mathrm{MgCl}_{2}$ Tris- $\mathrm{HCl}$ buffer solution was used to culture them at room temperature overnight, and then they were cultured for $2 \mathrm{~h}$ under the environment of ALP staining solution at room temperature in the dark. Water was used to wash them for a few minutes, and then methyl green was employed to restain and conventional water was then used to seal the sections.

Western blot analysis. Lysate and $1 \%$ (V/V) phenylmethylsulfonyl fluoride, respectively, were added into the IVD vertebral bodies of 4 -week-old WT and $\mathrm{P} 27^{-/-}$mice [vertebral body weight: lysate and 1\% (V/V) phenylmethylsulfonyl fluoride weight $=1: 20$ ], and the solutions were mixed well, followed by centrifugation at $10,050 \mathrm{x}$ g for $15 \mathrm{~min}$ at $4^{\circ} \mathrm{C}$. The supernatant was absorbed and full-automatic microplate reader was used to detect the protein concentration. Additionally, $30 \mathrm{mg}$ protein sample was separated by lauryl sodium dodecyl sulphate-polyacrylamide gel (SDS-PAGE) electrophoresis. The protein was then transferred from the gel onto nitrocellulose membrane

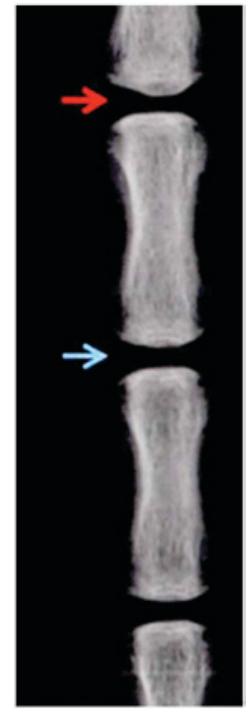

Figure 1. Intervertebral spaces of Co6-7 (red arrow) and Co7-8 (blue arrow) of mice in the WT group were normal at the end of the 4th week. WT, wild-type.

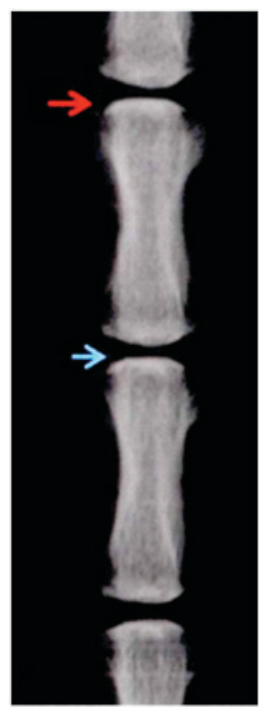

Figure 2. Intervertebral spaces of Co6-7 (red arrow) and Co7-8 (blue arrow) of mice in $\mathrm{P} 27^{-/}$group were obviously decreased at the end of the 4th week. $\mathrm{P} 27^{-/-}$, P27 gene knockout.

and developed via enhanced chemiluminescence, followed by X-ray film exposure and film-developing in a dark room. $\beta$-actin was served as internal control.

Statistical analysis. Statistical software, SPSS 20.0 (IBM, SPSS, Armonk, NY, USA), was employed for analysis. The measurement data were presented as mean \pm SD. The Chi-square test was used for label data and the paired sampled-test was utilized for the measurement data. For all the tests, a 5\% level of significance was used to draw the conclusions.

\section{Results}

$X$-ray examination. At the end of the fourth week of the model establishment, the average DHI\% of the WT group 


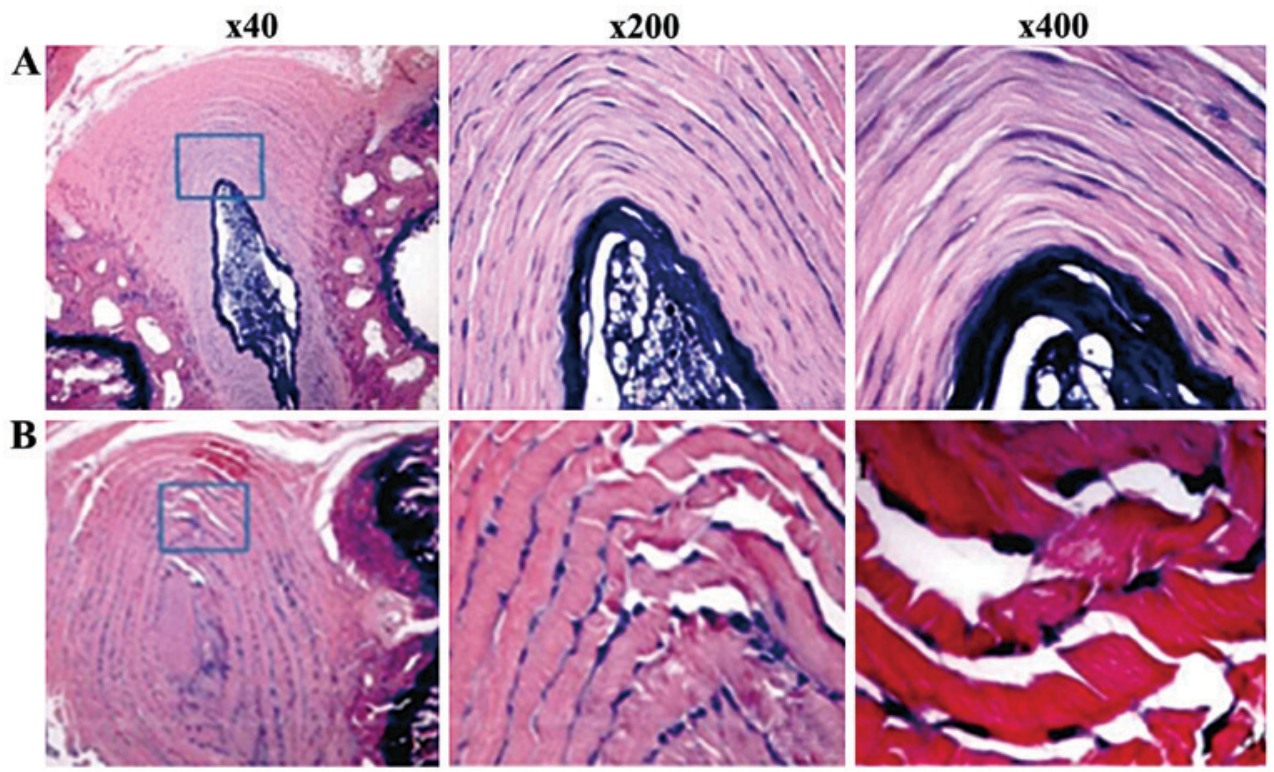

Figure 3. (A) Normal intervertebral disc staining in the WT group. (B) IVD staining in P27-/ group. WT, wild-type; IVD, intervertebral disc; P27 $7^{-/}$, P27 gene knockout.

A

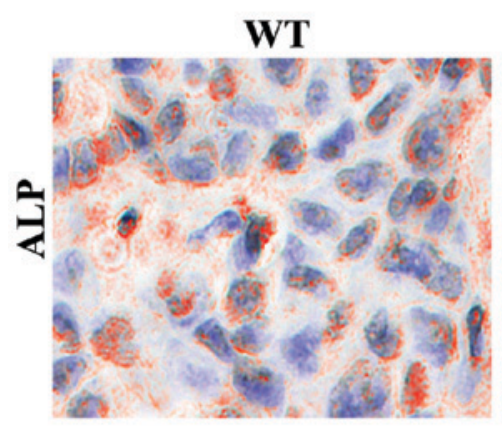

$\mathbf{P 2 7}{ }^{-/-}$

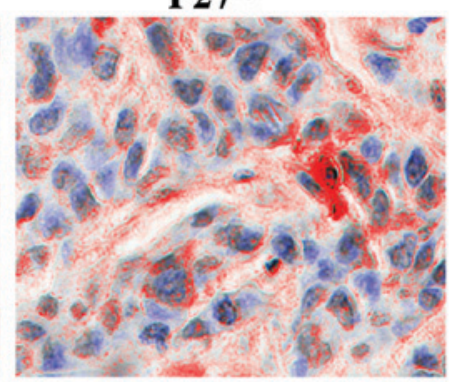

B

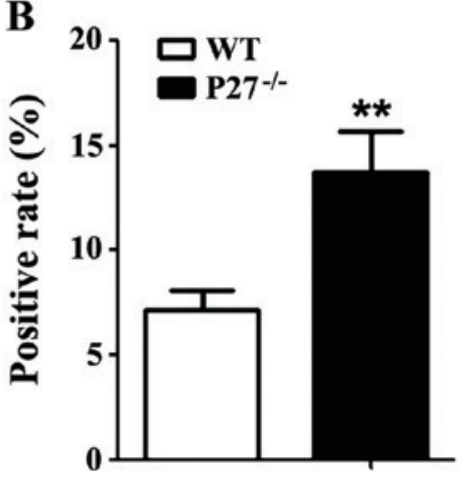

Figure 4. ALP histochemical staining result. (A) IVD ALP staining micrograph. (B) Columnar statistical figure of IVD ALP positive area rate. ALP, alkaline phosphatase; IVD, intervertebral disc. ${ }^{* *} \mathrm{P}<0.01$.

was $0.83 \pm 0.06$ but it was not statistically significant when compared with that at 0 week of the model establishment (P>0.05) (Fig. 1). At the end of the 4 th week since the model was established, the average DHI\% of the $\mathrm{P} 27^{-/}$group was $0.53 \pm 0.03$, indicating a significant difference when compared with the average $\mathrm{DHI} \%$ of $\mathrm{P} 27^{-/-}$at 0 week since model establishment ( $\mathrm{P}>0.05)$ (Fig. 2).

Result of histology. In the WT group, normal IVD staining indicated that round or elliptic nucleus pulposus tissue was evident. The collagen lamellae arrangement was normal and the boundary between nucleus pulposus and annulus fibrosus was clear. In addition, nucleus pulposus cell showed a star-like shape, and annulus fibrosus cells were fibroblast-like, which was located in the collagen fiberboard room (Fig. 3).

In the $\mathrm{P} 27^{-/}$group, mouse IVD staining indicated that puncturing side annulus fibrosus was fractured with needle-tip puncture trace. Furthermore, the interlamellar architecture of annulus fibrosus distributed in disorder, showing wavy-like shape and radial direction and concentric circle-like fracture image was evident. IVD nucleus pulposus was irregularly reduced to the small volume, extracellular matrix staining in nucleus pulposus became thin, and its boundary with annulus fibrosus was unclear (Fig. 4).

Influence of P27 deficiency on mouse IVD bone mass. ALP histochemical staining revealed that the ALP-positive area of mice in the $\mathrm{P} 27^{-/}$group was significantly increased compared with that of mice in the WT group (Fig. 4A and B). In addition, where Col-I immumohistochemical staining revealed that Col-I-positive area of mice in the $\mathrm{P} 27^{-/-}$group was apparently increased compared with that of mice in the WT group (Fig. 5A and B).

Influence of P27 deficiency on the Shh-signal pathway. The statistical significant increase in Smo-positive cell rate of mice in the $\mathrm{P} 27^{-/}$group was found compared to mice in the WT group (Fig. 6A and B). In order to further observe the influence of P27 deficiency on the changes of Shh-signal pathway, western blot analysis was used to detect the changes in the protein expression levels of Shh, Patched, Smoothened and Gli2 in IVD. It was found that the protein expression 
A

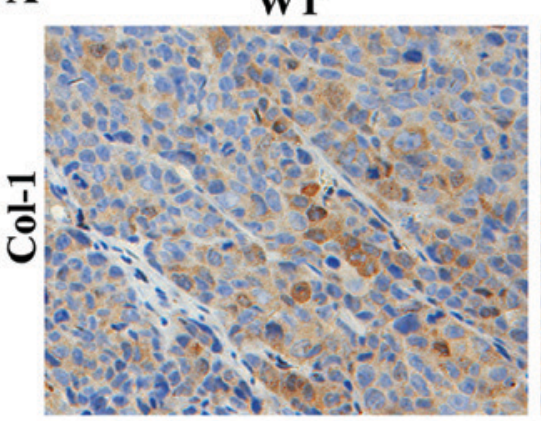

P27-

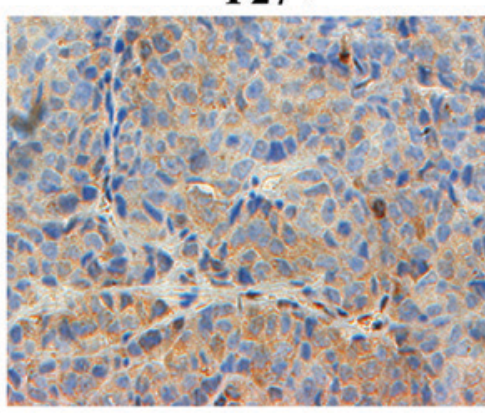

B

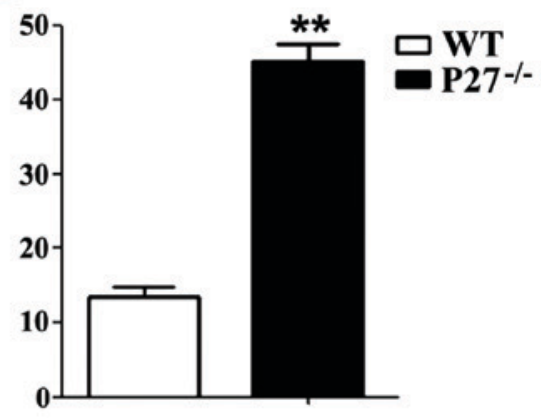

Figure 5. Col-I immumohistochemical staining result. (A) IVD Col-I staining micrograph (B) Columnar statistical figure of intervertebral disc Col-I positive area rate. IVD, intervertebral disc.** $\mathrm{P}<0.01$.

A

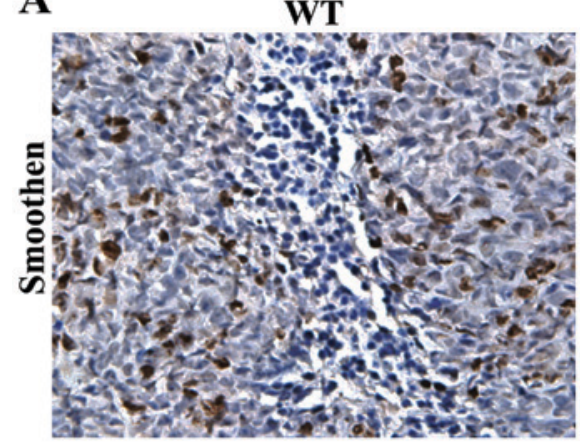

B

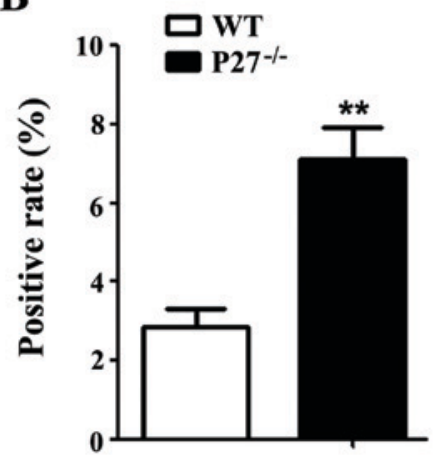

$\mathbf{P 2 7} \%$

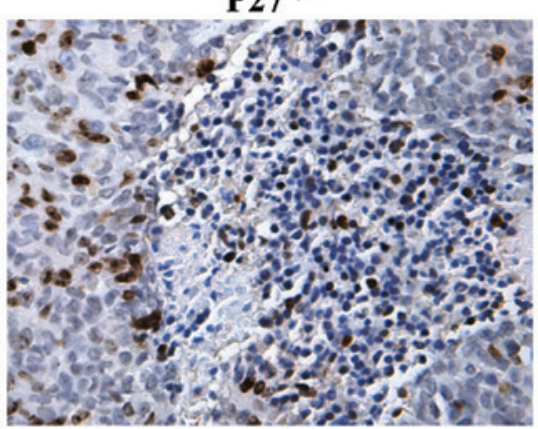

C

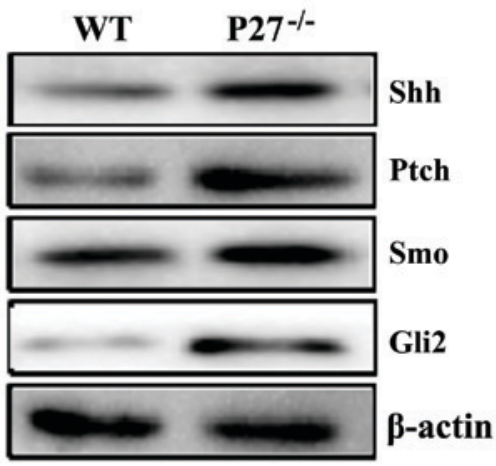

Figure 6. Shh-signal pathway expression of mice in the two groups. (A) IVD Smoothened staining micrograph. (B) Columnar statistical figure of Smo-positive cell rate. (C) Western blot analysis result of protein expression levels of Shh, Patched, Smoothened and Gli2 in IVD. IVD, intervertebral disc.* $<<0.01$.

levels of Shh, Patched, Smoothened and Gli2 of mice in the $\mathrm{P} 27^{-/}$group were markedly increased compared to mice in the WT group (Fig. 6C).

\section{Discussion}

The Shh-signal pathway is one of the Hedgehog pathways that regulate the development and differentiation of vertebrate entoderm, which is a highly conservative morphogenesis pathway of the medial axis organ development existing in both Drosophila melanogaster and vertebrate (10-13). Membrane proteins Ptch and Smo control Shh-signal transmission towards the cell, and receptor Ptch negatively regulates the Shh-signaling pathway (14-16). Receptor Smo is coded by proto-oncogene Smoothened which is homologous with G-protein-coupled receptor and consists of a single peptide chain of seven transmembrane regions, whereas the Ptch affects its function. Transcription factors of Shh-signal pathway belong to the Gli gene family, of which Glil, Gli2 and G1i3, with zinc finger, have been identified (17-20).

Previous findings have shown that Shh can induce notochord and apical plate cells into the IVD and bony vertebral body in the process of mouse embryonic development. However, when Shh is deficient, notochord and apical plate cells cannot normally develop into the IVD and bony vertebral body (21). This shows that Shh-signal pathway plays a key role in the development process of the IVD. It has been shown that the IVD nucleus pulposus cell can also excrete Shh in a 4-day-old mouse, and the activated Shh-signal pathway can thus interact with signal pathways, including TGF- $\beta$, BMP 
and Wnt, as well as regulate the expression of transcription factors, such as P27, SOX9, type-I collagen protein, type-II collagen protein and chondroitin sulfate, and as a result, regulate the growth and development of IVD (22). This finding indicates that the Shh-signal pathway is involved in the whole process of IVD occurrence and may play a crucial role in it. We also found that the protein expression levels of Shh, Patched, Smoothened and Gli2 of mice in the P27/- group were increased compared to those of mice in the WT group, suggesting that P27 deficiency can activate the Shh-signaling pathway.

ALP belongs to a critical enzyme in the process of osteoblast differentiation and can be used to measure osteoblast activity, whereas type-I collagen belongs to osteoblast product, and can be used to evaluate the osteoblast-differentiated degree. In the present study, ALP histochemical staining demonstrated that the ALP-positive area of mice in the P27 ${ }^{-/}$group was significantly increased compared to mice in the WT group. Furthermore, the Col-I immunohistochemical staining showed that the Col-I-positive area of mice in the $\mathrm{P} 27^{-/-}$group was significantly increased compared to mice in the WT group. These results indicate that P27 deficiency can induce an increase in osteoblast bone formation in the intervertebral disc.

In conclusion, P27 deficiency activates the expression of the Shh-signal pathway and promotes the proliferation of osteoblast. This plays a role in promoting IVD degeneration, which provides a scientific and reliable experimental basis for the treatment of IVD degeneration-related diseases in clinical practice.

\section{References}

1. DeLucca JF, Cortes DH, Jacobs NT, Vresilovic EJ, Duncan RL and Elliott DM: Human cartilage endplate permeability varies with degeneration and intervertebral disc site. J Biomech 49: 550-557, 2016

2. Chen HT, Huang AB, He YL, Bian J and Li HJ: Wnt11 overexpression promote adipose-derived stem cells differentiating to the nucleus pulposus-like phenotype. Eur Rev Med Pharmacol Sci 21: 1462-1470, 2017.

3. Cui YZ, Yang XH, Liu PF, Wang B and Chen WJ: Preliminary study on diagnosis of lumbar disc degeneration with magnetic resonance T1p, T2 mapping and DWI quantitative detection technologies. Eur Rev Med Pharmacol Sci 20: 3344-3350, 2016.

4. Ma T, Guo CJ, Zhao X, Wu L, Sun SX and Jin QH: The effect of curcumin on NF- $\kappa \mathrm{B}$ expression in rat with lumbar intervertebral disc degeneration. Eur Rev Med Pharmacol Sci 19: 1305-1314, 2015.

5. Liu C, Fei HD, Sun ZY and Tian JW: Bioinformatic analysis of the microarray gene expression profile in degenerative intervertebral disc cells exposed to TNF- $\alpha$. Eur Rev Med Pharmacol Sci 19 3332-3339, 2015.

6. Baranto A, Ekström L, Holm S, Hellström M, Hansson HA and Swärd L: Vertebral fractures and separations of endplates after traumatic loading of adolescent porcine spines with experimentally-induced disc degeneration. Clin Biomech (Bristol, Avon) 20: 1046-1054, 2005.
7. Lundin O, Ekström L, Hellström M, Holm S and Swärd L: Exposure of the porcine spine to mechanical compression: Differences in injury pattern between adolescents and adults. Eur Spine J 9: 466-471, 2000.

8. Mackiewicz Z, Salo J, Konttinen YT, Kaigle Holm A, Indahl A, Pajarinen $\mathrm{J}$ and Holm S: Receptor activator of nuclear factor kappa B ligand in an experimental intervertebral disc degeneration. Clin Exp Rheumatol 27: 299-306, 2009.

9. Shimura-Miura H, Hattori N, Kang D, Miyako K, Nakabeppu Y and Mizuno Y: Increased 8-oxo-dGTPase in the mitochondria of substantia nigral neurons in Parkinson's disease. Ann Neurol 46: 920-924, 1999.

10. Bach FC, Zhang Y, Miranda-Bedate A, Verdonschot LC, Bergknut N, Creemers LB, Ito K, Sakai D, Chan D, Meij BP and Tryfonidou MA: Increased caveolin-1 in intervertebral disc degeneration facilitates repair. Arthritis Res Ther 18: 59, 2016.

11. Thoreson O, Baranto A, Ekström L, Holm S, Hellström M and Swärd L: The immediate effect of repeated loading on the compressive strength of young porcine lumbar spine. Knee Surg Sports Traumatol Arthrosc 18: 694-701, 2010.

12. Arpinar VE, Rand SD, Klein AP, Maiman DJ and Muftuler LT: Changes in perfusion and diffusion in the endplate regions of degenerating intervertebral discs: A DCE-MRI study. Eur Spine J 24: 2458-2467, 2015

13. Wang B, Wang D, Yan T and Yuan H: MiR-138-5p promotes TNF- $\alpha$-induced apoptosis in human intervertebral disc degeneration by targeting SIRT1 through PTEN/PI3K/Akt signaling. Exp Cell Res 345: 199-205, 2016.

14. Jang TW, Ahn YS, Byun J, Lee JI, Kim KH, Kim Y, Song HS, Lee CG, Kwon YJ, Yoon JH and Jeong K: Lumbar intervertebral disc degeneration and related factors in Korean firefighters. BMJ Open 6: e011587, 2016.

15. Qin C, Zhang B, Zhang L, Zhang Z, Wang L, Tang L, Li S, Yang Y, Yang F, Zhang P and Yang B: MyD88-dependent Toll-like receptor 4 signal pathway in intervertebral disc degeneration. Exp Ther Med 12: 611-618, 2016.

16. Yang H, Yuan C, Wu C, Qian J, Shi Q, Li X, Zhu X and Zou J: The role of TGF- $\beta 1 / \mathrm{Smad} 2 / 3$ pathway in platelet-rich plasma in retarding intervertebral disc degeneration. J Cell Mol Med 20: 1542-1549, 2016

17. Nicole W, Tellegen AR, Niklas B, Creemers LB, Jeannette W, Christian F, Benz K, Grinwis GCM, Tryfonidou MA and Meij BP: Inflammatory profiles in canine intervertebral disc degeneration. BMC Vet Res 12: 1-12, 2016.

18. Zhang F, Zhao X, Shen H and Zhang C: Molecular mechanisms of cell death in intervertebral disc degeneration (Review). Int J Mol Med 37: 1439-1448, 2016.

19. Lv FJ, Peng Y, Lim FL, Sun Y, Lv M, Zhou L, Wang H, Zheng Z, Cheung KM and Leung VY: Matrix metalloproteinase 12 is an indicator of intervertebral disc degeneration co-expressed with fibrotic markers. Osteoarthritis Cartilage 24: 1826-1836, 2016.

20. Luo Y, Zhang L, Wang WY, Hu QF, Song HP and Zhang YZ: The inhibitory effect of salmon calcitonin on intervertebral disc degeneration in an ovariectomized rat model. Eur Spine J 24: 1691-1701, 2015.

21. Choi KS, Cohn MJ and Harfe BD: Identification of nucleus pulposus precursor cells and notochordal remnants in the mouse: Implications for disk degeneration and chordoma formation. Dev Dyn 237: 3953-3958, 2008.

22. Dahia CL, Mahoney E and Wylie C: Shh signaling from the nucleus pulposus is required for the postnatal growth and differentiation of the mouse intervertebral disc. PLoS One 7: e35944, 2012. 